\title{
La Terapia Dialéctico-Comportamental en el Sistema Nacional de Salud es posible: un caso clínico.
}

The Dialectical Behavior Therapy in the National Health System is possible: a clinical case.

\author{
Cristina Montero Fernández a, Jesús Pol Rodríguez a , Ione Esquer Terrazas a, Verónica Mañanes \\ Barral ${ }^{\text {b. }}$ \\ ${ }^{a}$ Residente de Psicología Clínica. Complejo Asistencial Universitario de Salamanca, España. ${ }^{b}$ Psicóloga . \\ Correspondencia: Cristina Montero Fernández,(monterofernandez.cristina@gmail.com)
}

Recibido: 30/12/2012; aceptado: 20/05/2013

\begin{abstract}
RESUMEN: El presente trabajo muestra el caso clínico de una mujer de 32 años con Trastorno Límite de Personalidad (TLP) con gran interferencia tanto en sus relaciones sociales y familiares como en su rutina diaria. Para la explicación del trastorno se integra el modelo de Linehan con el modelo de vulnerabilidadestrés y las teorías del apego, y en su tratamiento se utiliza el abordaje de las áreas que propone la Terapia Dialéctica Comportamental desde distintas Unidades de la red de Salud Mental. En un seguimiento de 9 meses la paciente se encuentra estable, ha mejorado en el ámbito interpersonal y ha iniciado una actividad laboral. Con este artículo ejemplificamos una manera de adaptar un tratamiento efectivo para el TLP, la Terapia Dialéctica Comportamental, al ámbito público sanitario a través de la coordinación de diferentes dispositivos. La efectividad conseguida nos lleva a reclamar estudios que avalen este planteamiento con el fin de aumentar la eficacia del tratamiento del TLP en el Sistema Público de Salud.
\end{abstract}

PALABRAS CLAVE: Trastorno Límite de Personalidad. Prestación de Atención de Salud. Psicoterapia.
ABSTRACT:This article shows the clinical case of a 32 years old woman with Borderline Personality Disorder (BPD), both high-interference in her social and family relationships as in her daily routine. For an explanation of the disorder, Linehan's model has been integrated with the vulnerability-stress model and attachment theories, and the treatment uses the Dialectical Behavior Therapy areas from different units of the Mental Health Network. In a 9-month follow-up the patient is stable, has improved her relation-ships and has initiated a work activity. With this article we exemplify a way to adapt an effective treatment for BPD, the Dialectical Behavior Therapy, to the Public Health System through the coordination of different devices. The effectiveness obtained leads to claim studies addressing this approach to increase the effectiveness of treatment of BPD in the Public Health System.

KEY WORDS: Borderline Personality Disorder. Delivery of Health Care. Psychotherapy. 


\section{Introducción.}

Según el DSM-IV-TR (1) la característica esencial del Trastorno Límite de Personalidad (TLP) es un patrón general de inestabilidad en las relaciones interpersonales, la autoimagen y la afectividad y una notable impulsividad. Éste supone actualmente un importante reto por varias razones: a) Su alta prevalencia, entre un 1-2\% en la población general, un $11 \%$ de los pacientes psiquiátricos ambulatorios y un $20 \%$ de los pacientes psiquiátricos ingresados (1-2), siendo el trastorno de personalidad (TP) más prevalente entre las personas atendidas en la red de salud mental pública estatal (3). b) Su alta comorbilidad con otros trastornos mentales, principalmente trastornos del estado de ánimo, trastornos relacionados con el consumo de sustancias, trastornos de la conducta alimentaria, trastorno de estrés postraumático y otros trastornos de ansiedad (4). c) Su gravedad, puesto que las conductas autolesivas y los intentos de suicidio son frecuentes en los individuos con este trastorno. Entre un 70-75\% han realizado al menos un acto de autolesión (5) y alrededor de un $10 \%$ de los casos acaban suicidándose $(1,6)$. Y d) Las dificultades en el manejo de estos pacientes en la práctica clínica dadas las propias características del trastorno (inestabilidad, impulsividad...) y la complicada contratransferencia que suelen generar en el profesional. Además, el pronóstico incierto y el que en muchas ocasiones necesiten un tratamiento de manera crónica lleva al agotamiento de recursos y unidades específicas a las que derivar al paciente y al burnout del terapeuta. Según Millon (7-8), "padre" de los trastornos de personalidad, éstos deberían ser considerados el centro mismo de la Psicopatología entera, constituyendo el origen y el contexto de los síndromes clínicos, cuyo carácter psicopatológico reside en una tendencia a generar conflictos intra e interpersonales y a no resolverlos. Dentro de los TP Millon considera al TLP uno de los más graves y disfuncionales, junto con el paranoide y el esquizotípico. Todos estos motivos hacen que sea importante seguir investigando en el tratamiento del TLP.

A pesar de que en la actualidad se acepta que el tratamiento de elección para los pacientes con TLP es la psicoterapia (9), necesitamos contar con más datos acerca de la eficacia de un tratamiento integral del trastorno y que pueda llevarse a cabo en la red pública sanitaria, con las adaptaciones que ello conlleva. En este sentido, uno de los programas que ha recibido mayor apoyo empírico es la Terapia Dialéctico-Comportamental (Dialectical Behavior Therapy, DBT) desarrollada por la Dra. Marsha M. Linehan y su grupo de investigación en la Universidad de Washington (Estados Unidos). Desde la aproximación biosocial de Linehan (10-12) el TLP se conceptualiza como una disfunción del sistema de regulación emocional producto de la interacción entre aspectos biológicos y un ambiente invalidante. El individuo presentaría una alta vulnerabilidad emocional, de origen biológico, consistente en una alta sensibilidad ante los estímulos emocionales, una 
NOTAS CLÍNICAS

tendencia a experimentar emociones muy intensas y dificultades para retornar a su línea de base emocional. Además, tendría muchas dificultades para identificar, etiquetar y modular las emociones desarrollando un temor acusado a experimentarlas y, por tanto, utilizaría estrategias de evitación como las conductas autolesivas o el abuso de tóxicos. A su vez, el ambiente invalidante se refiere a un patrón de crianza que responde de manera inapropiada o no contingente a la comunicación de sentimientos, de modo que se le transmite al niño que su regulación emocional no es correcta, pero no se le enseña la manera adecuada de hacerlo. Este ambiente castiga la expresión de emociones negativas, pero refuerza intermitentemente las expresiones emocionales extremas, a la vez que envía mensajes erróneos sobre el control emocional y la solución de problemas. De esta forma la interacción entre una alta vulnerabilidad emocional y un ambiente invalidante produce inestabilidad emocional y las disfunciones constituyen intentos por controlar la emoción o por conseguir apoyo del medio, convirtiéndose los intentos de solución de la inestabilidad en los principales problemas.

A partir de este modelo teórico Linehan desarrolla un programa de tratamiento cuyo objetivo es que el paciente aprenda a modular la emocionalidad extrema, reduzca las conductas desadaptativas dependientes de la emoción y aprenda a validar sus emociones, pensamientos y comportamientos. La DBT se estructura en una fase de pretratamiento, dirigida a la orientación del paciente hacia la terapia (establecimiento de la relación terapéutica, las metas y los compromisos), y tres fases de tratamiento con metas ordenadas jerárquicamente. En la primera fase se trabaja la reducción de conductas suicidas, la reducción de conductas que interfieren en el proceso terapéutico, la reducción de conductas que interfieren en la calidad de vida del paciente y el incremento de habilidades (toma de conciencia o mindfulness, regulación emocional, tolerancia al malestar y habilidades sociales); en la segunda, el tratamiento del estrés postraumático y en la tercera, el logro de metas individuales. En la DBT se combina la terapia individual con la grupal y las estrategias básicas son la validación y la solución de problemas. Cuenta con avales empíricos suficientes como para ser considerado un tratamiento psicológico probablemente eficaz para el TLP (13).

Por último, mencionar la importancia del modelo de vulnerabilidad-estrés, bien establecido en el estudio de la esquizofrenia (14) pero aplicable también a otros trastornos mentales como los de personalidad. El diagnóstico de TP no implica una disfuncionalidad constante, de modo que muchos pacientes mantienen cierta estabilidad hasta que determinado estresor provoca que entren en "crisis" intensificándose los rasgos de personalidad desadaptativos y apareciendo el deterioro en distintas áreas vitales y el malestar. Dentro de esta vulnerabilidad no podemos olvidar los estudios sobre el apego (15-19) que postulan que un apego inseguro, tanto evitativo como ambivalente, podría predisponer al desarrollo de un 
TLP en la edad adulta. Una primera relación de apego inestable y caótica donde las necesidades afectivas del niño son atendidas de manera irregular, podría generar un patrón de relaciones interpersonales inestables e intensas, esfuerzos frenéticos por evitar el abandono, temor a estar solo o ser rechazado y confusión y variabilidad en torno a la imagen de sí mismo. Como el patrón de autorregulación que hubiera derivado de un apego seguro no ha podido establecerse, la regulación ha de venir del exterior bien en forma de ayuda y dependencia, bien por medios diferentes como conductas impulsivas y el uso de drogas, alcohol o fármacos que disminuyan el malestar.

A continuación se expone el caso de una paciente diagnosticada de TLP cuyo trastorno puede explicarse mediante la integración de los modelos aquí descritos: el de Linehan, el modelo de vulnerabilidad-estrés y las teorías del apego. El objetivo de este trabajo es presentar la evolución de dicha paciente a partir del tratamiento integral llevado a cabo en distintos dispositivos de la red pública de Salud Mental, abordando las áreas de tratamiento que plantea la DBT.

\section{Identificación del paciente, evaluación y formulación clínica del caso.}

R. es una mujer de 32 años, la mayor de dos hermanos, soltera y sin hijos. Licenciada en Derecho. Sus padres se divorciaron cuando ella tenía 12 años. Su madre falleció por una cardiopatía en 2010, a los 51 años de edad. R. vive sola en un entorno rural desde el verano de 2011, a escasa distancia de su padre y la mujer de éste y sustentada por ellos. Ha desempeñado diversos empleos temporales, aunque de corta duración: en Atención al Cliente en una oficina de Correos, como comercial, abogada en un bufete... Muestra antecedentes de patología ansiosodepresiva desde 2006, siendo evaluada y tratada por distintos Psicólogos y Psiquiatras en varias ciudades (Barcelona, Madrid, Salamanca) y recibiendo diversos diagnósticos: trastorno depresivo mayor, trastorno distímico, trastorno adaptativo mixto ansioso-depresivo, trastorno de ansiedad generalizada y trastorno de la personalidad. Ha seguido diferentes tratamientos psicofarmacológicos con antidepresivos, estabilizadores, benzodiacepinas y neurolépticos con escasa o nula respuesta a todos ellos, cumplimiento irregular y aparición de múltiples efectos secundarios. Todo ello, según refiere la paciente, le ha impedido hacer "una vida normal" tanto en el aspecto laboral como en las relaciones familiares y sociales, necesitando constantemente el apoyo y la ayuda de su familia.

En los últimos años de la biografía de R. encontramos diversos momentos de estrés donde entra en contacto con los servicios de Salud Mental. Tras licenciarse en Derecho en 2003, desempeñar diversos trabajos y vivir de una manera 
NOTAS CLÍNICAS

autónoma, en 2005 rompe una relación sentimental de 9 años de duración y se muda a Barcelona con su madre, afectada de alcoholismo, para cuidarla. En 2006 es diagnosticada de Episodio Depresivo Mayor, coincidiendo con la preparación de oposiciones, el cuidado de su madre y cierto aislamiento social. R. acude al mismo Psiquiatra que su madre y a las terapias de grupo de Alcohólicos Rehabilitados. En ese momento aparecen ideas autolíticas que ella misma define como "pensamientos autodestructivos patológicos". En 2008 se traslada a vivir a Salamanca con su madre y la pareja de ésta. Acude a su ESM por un posible cuadro hipomaníaco desencadenado por antidepresivos y abuso de café para paliar los efectos de las benzodiacepinas, de las que también abusa. Presenta asimismo clínica ansiosodepresiva. En 2009 mantiene una relación sentimental con la pareja de su madre y desde entonces padece frecuentes crisis de ansiedad y presenta un estado de ánimo disfórico, labilidad emocional, verborrea, pensamiento saltígrado, disminución de la concentración, desorganización de horarios con tendencia a la clinofilia y aislamiento social. Es derivada al Hospital de Día (HD), donde se le diagnostica de Trastorno Depresivo Mayor, intoxicación por cafeína y rasgos de dependencia y compulsividad en el Eje II, así como problemas con el grupo primario de apoyo en el Eje IV. En ese momento presenta importantes problemas relacionales con su madre, en el contexto de una relación ambivalente. En el momento del alta se encuentra más estable y activa, ha organizado horarios, retomado relaciones sociales y empieza a responsabilizarse de las tareas del hogar. Ha mejorado el control sobre el consumo de café y la toma de la medicación y tiene proyectos de iniciar un curso. En 2010 se traslada a vivir a Madrid y allí comienza su "peregrinaje" por distintos Psiquiatras y Psicólogos. En abril fallece su madre, con lo que aumenta la clínica ansioso-depresiva y, con ella, el abuso de benzodiacepinas y café así como las visitas a distintos profesionales. También emplea todo el dinero de la herencia de su madre en compras innecesarias. Refiere la paciente que "no lo hacía en un estado de euforia, sino sintiéndose previamente mal, ansiosa, vacía y triste, y así se distraía". En el verano de 2011, tras gastar todo su dinero y ser despedida de distintos trabajos, se traslada a vivir a su pueblo de origen, a un piso propiedad de la mujer de su padre. Es atendida en su Equipo de Salud Mental (ESM), tanto por Psicología Clínica como por Psiquiatría, donde se le pauta tratamiento con Escitalopram $15 \mathrm{mg}$. una vez al día y Lormetazepam $2 \mathrm{mg}$. al día, con incremento por su parte de las dosis de benzodiacepinas prescritas. Acude simultáneamente a una Psicóloga privada y, por último, consulta con la Comunidad de Proyecto Hombre desde donde es derivada a la Unidad de Patología Dual (UPD) para desintoxicación de benzodiacepinas, estabilización psicopatológica y clarificación diagnóstica.

Al inicio del programa de tratamiento en la UPD se le aplican diversas pruebas psicométricas: IPDE, EPI, 16-PF, SCL-90-R, MCMI-II, STAI y WAIS-III. En ellas aparece un patrón de personalidad límite con marcados rasgos dependientes, 
histriónicos, obsesivos y autodestructivos. Destacan las elevadas puntuaciones en ansiedad y distimia, así como en somatización. En este momento se formula una hipótesis explicativa del TLP y los síntomas asociados congruente con el modelo de Linehan, con el modelo de vulnerabilidad-estrés y con las teorías del apego. La aceptación de esta hipótesis por los distintos dispositivos que traten a R. y la aplicación de las técnicas a ella asociadas será lo que marque la mejoría sintomatológica de la paciente. Dicha hipótesis puede describirse como sigue: Tras un funcionamiento previo aparentemente adaptado, aparece un acusado malestar en R. y un deterioro importante en las áreas social, familiar y laboral. La relación conflictiva entre sus padres, que termina con la separación de éstos y el matrimonio del padre con otra mujer, una madre con tendencia al ánimo depresivo y el abuso de alcohol, un refuerzo intermitente de las expresiones emocionales de R. y posibles factores hereditarios, se constituyen en aspectos de vulnerabilidad. Además, en este contexto, son importantes el apego establecido con las figuras paternas y la falta de límites educativos claros y firmes: un padre que abandona a su familia por otra mujer y se siente culpable, con lo que concede cualquier capricho a sus hijos para no perder su cariño, aunque los "desatiende" a nivel emocional; y una madre con problemas psicológicos que mantiene una relación ambivalente y dependiente con ellos. Con su madre fallecida, la paciente realiza una búsqueda de la relación con su padre de una manera primitiva y pueril. Parece que en la vida de R. la canalización de sus rasgos obsesivos hacia los estudios y el mantenimiento de una pareja estable como figura de apego y sostén emocional constituían factores protectores que al desaparecer y unirse a determinadas circunstancias estresantes (cuidado de su madre enferma, fracaso en oposiciones y empleos, pérdida de relaciones interpersonales, duelo por la muerte de la madre, vuelta al pueblo y fracaso en su independencia...) supusieron una ruptura en su biografía y una exacerbación de sus rasgos patológicos de personalidad, con el consiguiente surgimiento de síntomas ansioso-depresivos, abuso de fármacos y deterioro en sus relaciones y funcionamiento.

\section{Tratamiento y evolución.}

Durante su ingreso en la UPD, R. refiere su historia y sus síntomas de ansiedad con gran dramatismo y múltiples quejas en relación con la ansiedad de tipo dolor, ahogo, temblores, imposibilidad para caminar, ver o hablar..., síntomas que explica de forma vaga e inconcreta y que motivan por su parte constantes demandas de atención a todo el personal de la Unidad, de medicación y de medidas especiales. No presenta síntomas de tipo psicótico ni ideas de muerte o suicidio y su estado de ánimo es muy variable y condicionado por la clínica referida y la res- 
NOTAS CLÍNICAS

puesta del entorno a sus peticiones. En las entrevistas muestra una actitud teatral e histriónica, aunque se encuentra bien integrada en el grupo de pacientes y establece rápidamente relaciones. En las entrevistas familiares se muestra manipuladora; el padre de R. y su mujer aparecen desbordados por sus continuas quejas, demandas y chantajes, y la definen como "mentirosa, egocéntrica, caprichosa, culpa a los demás de sus problemas, autoexigente y poco tolerante con los demás, manipuladora, con continuas llamadas de atención, elude responsabilidades y no tiene una rutina ni hábitos "normales", por ejemplo, tiene la casa hecha un desastre". La esposa del padre cree que éste "no ha sabido ponerle límites porque se sentía culpable por haberles abandonado" y por ello permite que R. le manipule y que interfiera en su relación de pareja, "como ya hizo en la relación sentimental de su madre".

El diagnóstico en la UPD, según DSM-IV-TR, es el siguiente: En el Eje I: F.13.1 Abuso de benzodiacepinas (305.40), en Eje II: F60.31 Trastorno límite de la personalidad (301.83), sin diagnóstico en el Eje III, en el Eje IV: Vive sola, pero sustentada por su padre; fallecimiento reciente de la madre; desempleo; y en el Eje V: EEAG de 45. El plan farmacológico que se estableció fue: Sertralina 100 mg. una vez al día, Pregabalina $300 \mathrm{mg}$. dos veces al día, Olanzapina $5 \mathrm{mg}$. dos veces al día y Mirtazapina $30 \mathrm{mg}$. una vez al día. El tratamiento psicoterapéutico consistió en contención y apoyo psicológico a la desintoxicación física, entrevistas individuales y familiares con el fin de explorar y establecer expectativas realistas y planes de futuro, terapia de grupo, explicación de su trastorno de personalidad e inicio de entrenamiento en solución de problemas y toma de decisiones como alternativa a la impulsividad y el abuso de sustancias.

Tras dos meses ingresada en la UPD es derivada al HD (aunque tres días antes de la fecha de alta programada pide el alta voluntaria). Durante su estancia recibe el tratamiento farmacológico pautado previamente en la UPD, asiste a terapia de grupo, realiza entrevistas individuales y familiares y lleva a cabo las actividades propias del HD, donde se incluye un programa de Mindfulness, un módulo de Entrenamiento en Solución de Problemas y un módulo de Entrenamiento en Habilidades Sociales (con especial énfasis en la conducta asertiva). Al alta continúa estable, menos ansiosa, más activa, ha organizado horarios, se concentra mejor, se ocupa de tareas domésticas y ha iniciado un voluntariado y la búsqueda de empleo. La EEAG al ingreso es de 45 y de 70 al alta y la HoNOS, de 31/48 al ingreso y de $11 / 48$ al alta.

Como podemos observar, el tratamiento llevado a cabo cubre las áreas propuestas por la DBT:

-Fase de pretratamiento: Abordada en la UPD y en las entrevistas iniciales del HD.

-Fase de tratamiento: reducción de las conductas que interferían en la tera- 
pia (búsqueda constante de atención y abandono de los tratamientos), de las que interferían en la calidad de vida (consumo de benzodiacepinas, peregrinaje por distintos especialistas, compras, quejas, cuidado del hogar...) y aumento de habilidades comportamentales. Para ello se utiliza: mindfulness (en HD), habilidades de tolerancia al malestar: fomentar la aceptación del sufrimiento (en UPD y HD), habilidades de regulación emocional (UPD y HD), habilidades de eficacia interpersonal (terapia grupal en UPD y, especialmente, en HD), módulo de solución de problemas (tarea iniciada en UPD y entrenada con profundidad en HD) y módulo de entrenamiento en asertividad (HD). Las tareas pendientes serían el tratamiento del estrés postraumático (elaboración de acontecimientos traumáticos o pérdidas, como la separación de los padres, de su novio o la muerte de la madre) y el logro de metas individuales (como la independencia y el mantenimiento de un empleo), que son las que se están trabajando actualmente a nivel individual en su ESM.

\section{Resultados.}

Con el tratamiento en UPD y HD, a partir de la hipótesis explicada y abordando las fases de la DBT, se ha conseguido la desintoxicación de benzodiacepinas, la estabilidad psicopatológica de la paciente, un mayor distanciamiento de los síntomas, organización de horarios, la asunción de responsabilidades y una mayor autonomía, disminuyendo las conductas dependientes y de llamada de atención. Tras finalizar el tratamiento en HD, con una duración de un mes y medio, la paciente es remitida de nuevo a su ESM donde realiza un seguimiento por Psiquiatría y Psicología Clínica. A los 9 meses de seguimiento R. ha mejorado sus relaciones familiares, aunque las relaciones sociales siguen siendo escasas. Refiere que ha aprendido a canalizar su ansiedad y a convivir con ella. Se encuentra más estable anímicamente y lleva 6 meses trabajando como comercial.

\section{Discusión}

La DBT ha mostrado su efectividad en el contexto clínico (20) a pesar de ser considerada un tratamiento probablemente eficaz para el TLP en la literatura científica (13). No obstante, faltan estudios que adapten este programa al Sistema Público de Salud, así como otros que avalen la eficacia del tratamiento integral (psicoterapéutico y psicofarmacológico) del TLP. Con el artículo presentado hemos pretendido ejemplificar un modo de realizar la DBT en el ámbito público sanitario 
a través de la coordinación de distintos dispositivos, mediante el abordaje individual y grupal de las áreas de tratamiento que propone Linehan. En nuestro Sistema Sanitario no disponemos actualmente de un gran número de unidades que realicen tratamientos especializados para el TLP (21), por ello adecuar los programas efectivos a los recursos con los que contamos en cada momento supondría un gran avance en el tratamiento de este trastorno. Obviamente, en nuestro trabajo existen las limitaciones metodológicas propias del diseño AB con un solo sujeto, siendo difícilmente generalizable la efectividad a otros pacientes; por esto sería interesante realizar estudios controlados adicionales que avalen la eficacia del abordaje de las áreas planteadas por la DBT en diferentes dispositivos de la red de Salud Mental, de forma que adaptemos esta terapia al momento en que recibimos al paciente, al lugar en que lo hacemos y a los recursos de los que disponemos.

\section{BIBLIOGRAFÍA:}

(1) American Psychiatric Association. DSM-IV-TR. Manual Diagnóstico y Estadístico de los Trastornos Mentales: Texto Revisado. Barcelona: Elsevier Masson, 2002.

(2) Selva G, Bellver F y Carabal. Epidemiología del trastorno límite de la personalidad. En Cervera G, Haro G y Martínez-Raga J (Ed.). Trastorno límite de la personalidad. Paradigma de la comorbilidad psiquiátrica. Madrid: Editorial Médica Panamericana, 2005.

(3) Lana F, Fernández San Martín MI, Sánchez Gil C y Bonet E. Study of personality disorders and the use of services in the clinical population attended in the mental health network of a community area. Actas Esp Psiquiatr 2008; 36: 331-6.

(4) Girolano G y Dotto P. Epidemiology of personality disorders. En Gelder M, López-Ibor JJ y Andreasen N (Eds.). New Oxford Textbook of Psychiatry. Oxford: Oxford University Press, 2000.

(5) Cowdry RW, Picar D y Davis R. Symptoms and EEG findings in the borderline syndrome. Int J Psychiat Med 1985; 15, 201-11.

(6) Paris J y Zweig-Frank H. A 27 year follow-up of patients with borderline personality disorder. Comp Psychiat 2001; 42, 482-7.

(7) Millon T. Psicopatología moderna: Un enfoque biosocial de los aprendizajes erróneos y de los disfuncionalismos. Barcelona: Salvat, 1976.

(8) Millon T y Davis R. Trastornos de la personalidad. Más allá del DSM-IV. Barcelona: Masson, 1998.

(9) American Psychiatric Association. Practice guideline for the treatment of patients with borderline personality disorder. Am J Psychiatry 2001; 158: 1-52.

(10) Linehan MM. Manual de tratamiento de los Trastornos de Personalidad Límite. Barcelona: Paidós, 2003.

(11) García Palacios A. La Terapia Dialéctico-Comportamental. EduPsykhé 2006; 5 (2): 255-71.

(12) Aramburú B. La Terapia Dialéctica Conductual para el Trastorno Límite de la Personalidad. Psicología Conductual 1996; 4 (1): 123-40. 
(13) Quiroga E y Errasti JM. Tratamientos psicológicos eficaces para los trastornos de personalidad. Psicothema 2001; 13 (3): 393-406.

(14) Zubin J y Spring B. Vulnerabiliby. A new view of schizophrenia. J Abnorm Psychol 1977; $86,103-26$.

(15) Bakermans-Kranenburg MJ y Van Ijzendoorn MH. The first 10,000 Adult Attachment Interviews: distributions of adult attachment representations in clinical and non-clinical groups. Attach Hum Dev 2009; 11(3), 223-63.

(16) Bateman A y Fonagy P. The development of an attachment-based treatment program for borderline personality disorder. B Menninger Clin 2003; 67, 187-211.

(17) Crawford TN, Livesley WJ, Jang KL, Shaver, PR, Cohen P y Ganiban J. Insecure attachment and personality disorder: A twin study of adults. Eur J Personality 2007; 21, 191-208.

(18) Mosquera D y González A. El apego inseguro-ambivalente y sus efectos en el adulto con Trastorno Límite de la Personalidad. X Jornadas de Apego y Salud Mental. International Attachment Network. Madrid, 2009.

(19) Mosquera D y González A. Del apego temprano al TLP. Mente y Cerebro 2011; 46: 18-27.

(20) Kröger C, Schweiger U, Sipos V, Arnold R, Kahl KG, Rudolf S y Reinecker H. Effectiveness of dialectical behavior therapy for borderline personality disorder in an inpatient setting. Behav Res Ther 2006; 44: 1211-7.

(21) García MT, Martín MF y Otín R. Tratamiento integral del Trastorno Límite de Personalidad. Rev Asoc Esp Neuropsiq 2010; 30 (106), 263-78. 